\title{
Effective grain surface area in the formation of molecular hydrogen in interstellar clouds
}

\author{
S. K. Chakrabarti ${ }^{1,2}$, A. Das ${ }^{2}$, K. Acharyya ${ }^{2}$, and S. Chakrabarti2,3 \\ 1 S. N. Bose National Centre for Basic Sciences, Salt Lake, Kolkata, 700098, India \\ e-mail: chakraba@bose.res.in \\ 2 Centre for Space Physics, Chalantika 43, Garia Station Rd., Kolkata, 700084, India \\ e-mail: [ankan; acharyya]@csp.res.in \\ 3 Maharaja Manindra Chandra College, 20 Ramkanta Bose Street, Kolkata, 700003, India \\ e-mail: sonali@csp.res.in \\ Received 31 March 2006 / Accepted 6 June 2006
}

\section{ABSTRACT}

\begin{abstract}
Aims. In interstellar clouds, molecular hydrogens are formed from atomic hydrogen on grain surfaces. An atomic hydrogen hops around until it finds another one with which it combines. This necessarily implies that the average recombination time, or equivalently, the effective grain surface area depends on the relative numbers of atomic hydrogen influx rate and the number of sites on the grain. Our aim is to discover this dependency.

Methods. We perform a numerical simulation to study the recombination of hydrogen on grain surfaces in a variety of cloud conditions. We use a square lattice (with a periodic boundary condition) of various sizes on two types of grains, namely, amorphous carbon and olivine.

Results. We find that the steady state results of our simulation match very well with those obtained from a simpler analytical considerations provided the "effective" grain surface area is written as $\sim S^{\alpha}$, where $S$ is the actual physical grain area and $\alpha$ is a function of the flux of atomic hydrogen, which is determined from our simulation. We carry out the simulation for various astrophysically relevant accretion rates. For high accretion rates, small grains tend to become partly saturated with $\mathrm{H}$ and $\mathrm{H}_{2}$, and the subsequent accretion will be partly inhibited. For very low accretion rates, the number of sites to be swept before a molecular hydrogen can form is too large compared to the actual number of sites on the grain, implying that $\alpha$ is greater than unity.
\end{abstract}

Key words. ISM: molecules - astrochemistry - methods: numerical

\section{Introduction}

It has been long known that the formation of $\mathrm{H}_{2}$ in interstellar clouds takes place on the grain surfaces and then the molecules are released into the gas phase. Considerable studies have been made since then to understand the real physical processes that are taking place both theoretically (e.g., Hollenbach et al. 1971; Takahashi et al. 1999; Biham et al. 2001) and experimentally (e.g., Pirronello et al. 1999). A quantitative estimation based on physisorption of the formation rate of $\mathrm{H}_{2}$ was carried out by Biham et al. (2001) and Green et al. (2001). It was found that a significant production is possible in cooler $(\sim 10-25 \mathrm{~K})$ clouds (Rae et al. 2003). Cazaux \& Tielens (2002, 2004) use both physisorption and chemisorption to demonstrate that $\mathrm{H}_{2}$ production is possible at high temperatures $(\sim 200-400 \mathrm{~K})$ also. Recently, Acharyya et al. (2005) and Acharyya \& Chakrabarti (2005) computed the formation of $\mathrm{H}_{2}$ from $\mathrm{H}$, assuming various cloud conditions and using rate and master equations presented by Biham et al. (2001). If one considers only $\mathrm{H}$ and $\mathrm{H}_{2}$, then, in a steady state, the number of hydrogen atoms $\left(n_{\mathrm{H}}\right)$ and molecules $\left(n_{\mathrm{H} 2}\right)$ on a grain surface are obtained by equating the effective influx (or, accretion) rate $\phi_{\mathrm{H}}$ of $\mathrm{H}$ with the rate at which they are used up to form $\mathrm{H}_{2}$ and/or get desorbed from the grain surface into the gas phase, i.e.,

$\phi_{\mathrm{H}}=W_{\mathrm{H}} n_{\mathrm{H}}+2 a_{\mathrm{H}} n_{\mathrm{H}}^{2}$,

and

$W_{\mathrm{H} 2} n_{\mathrm{H} 2}=\mu a_{\mathrm{H}} n_{\mathrm{H}}^{2}$.
Here, $\phi_{\mathrm{H}}$ is the effective flux (in units of number/s) of $\mathrm{H}$ on the grain surface, $W_{\mathrm{H}}$ and $W_{\mathrm{H} 2}$ (both in units of $\mathrm{s}^{-1}$ ), are the desorption coefficients of $\mathrm{H}$ and $\mathrm{H}_{2}$ respectively, $a_{\mathrm{H}}$ (in units of $\mathrm{s}^{-1}$ ) is the effective rate of recombination of two $\mathrm{H}$ atoms to form one $\mathrm{H}_{2}$ molecule, and $1-\mu$ is the fraction of $\mathrm{H}_{2}$ formed on the grain that are spontaneously released to the gas phase. Solving these simple equations, we find in the steady state the number of $\mathrm{H}$ and $\mathrm{H}_{2}$ on a grain surface to be,

$$
\begin{aligned}
n_{\mathrm{H}} & =\frac{\sqrt{W_{\mathrm{H}}^{2}+8 a_{\mathrm{H}} \phi_{\mathrm{H}}}-W_{\mathrm{H}}}{4 a_{\mathrm{H}}}, \\
n_{\mathrm{H} 2} & =\frac{\mu a_{\mathrm{H}} n_{\mathrm{H}}^{2}}{W_{\mathrm{H}}} .
\end{aligned}
$$

We ignore $\mathrm{H}_{2}$ formation by tunneling since the experiments indicate hopping to be the major process (see Pironello et al. 1999; Katz et al. 1999). In the literature, it is customary to define the effective rate of recombination

$$
a_{\mathrm{H}}=A_{\mathrm{H}} / S,
$$

(see Acharyya et al. 2005, and references therein), where $A_{\mathrm{H}}$ is the hopping rate (inverse of the diffusion time $T_{\mathrm{d}}=1 / A_{\mathrm{H}}$ ) on a grain surface and $S$ is the number of sites on the grain surface (Biham et al. 2001). The argument for reducing the diffusion rate by a factor of $S$ is this: on an average, there are $n=S^{1 / 2}$ number of sites in each direction of the grain. Since the hopping 
is random, it would take a square of this number of hopping, namely, $n^{2}=S$, to reach a distance located at $n=S^{1 / 2}$ sites away, where, on an average, another $\mathrm{H}$ is available. Thus, the effective recombination rate was chosen to be $A_{\mathrm{H}} / S$. However, when the flux is very high in a small grain, many sites would be full and an $\mathrm{H}$ need not hop a distance of $S^{1 / 2}$ to meet another one. Similarly, when the accretion rate is too low, one $\mathrm{H}$ may have to sweep the whole grain several times before it can meet another $\mathrm{H}$ to recombine. Thus, it is expected that the effective rate of recombination would be a function of the number of sites, as well as the flux of $\mathrm{H}$ on the grain. If we assume that the effective number of sites could be written as $S^{\alpha_{0}}$, where $\alpha_{0}$ is a constant for a given flux and grain, then, in principle, we could define the effective recombination rate to be,

$a_{\mathrm{H}}=A_{\mathrm{H}} / S^{\alpha_{0}}$,

and we expect that the higher the accretion rate, the lesser the value of $\alpha_{0}$ would be. In the opposite limit, when the accretion rate is low, one would get $\alpha_{0}>1$, since the effective site number is higher than $S$. To see this effect, we clearly need to actually compute time dependences of $\mathrm{H}$ and $\mathrm{H}_{2}$ by numerical simulation and derive $\alpha_{0}$ when a "steady state" is reached. We do that in this paper.

In the next section, we present the logic based on which the simulation is carried out. In Sect. 3, we present our results under various grain conditions and compare our results with the expectation from the steady state (Eqs. $(2 a, b))$. We show that these two results match only if $\alpha_{0}$ is used in the steady state equations. Finally, in Sect. 4 we draw our conclusions.

\section{Numerical simulation}

The simulation is based on several physical processes might be taking place on the grain surface. We assume each grain to be square in shape having $S=n^{2}$ number of sites (where $n$ is the number of sites in each direction). We use the periodic boundary condition. We consider only the monolayer on the grain surface. Let $A_{\mathrm{H}}=v \exp \left(-E_{0} / k_{\mathrm{b}} T\right)$, the rate at which $\mathrm{H}$ hops from a site to the next, where $v$ is the vibrational frequency,

$v=\frac{2 s E_{\mathrm{d}}}{\pi^{2} m_{\mathrm{H}}} \mathrm{s}^{-1}$.

Here, $s \sim 10^{14} \mathrm{~cm}^{-2}$ is the surface density of sites on a grain, $m_{\mathrm{H}}$ is the mass (in gm) of the $\mathrm{H}$ atom, and $E_{\mathrm{d}}$ is the binding energy corresponding to physisorption (in $\mathrm{meV}$ ). We choose the minimum time step to be $1 / A_{\mathrm{H}}$, the hopping time, and advance the global time by this time step. Let $F_{\mathrm{H}}$ be the accretion rate of $\mathrm{H}$ which increases the number of $\mathrm{H}$ on a grain by sticking to it. Here, $F_{\mathrm{H}}=A\langle V\rangle N_{\mathrm{H} \mathrm{s}} \mathrm{s}^{-1}$, where $N_{\mathrm{H}}$ is the number density (in $\mathrm{cm}^{-3}$ ) of $\mathrm{H}$ in the gas phase, $\langle V\rangle$ (in $\mathrm{cm} / \mathrm{s}$ ) is the average thermal velocity of the particle in the gas phase, and $A$ is the physical grain surface area (in $\mathrm{cm}^{2}$ ); when $10^{2} \lesssim N_{\mathrm{H}} \lesssim 10^{7}$ and $V \sim 10^{4}$, we have $3 \times 10^{-8}<F_{\mathrm{H}}<3 \times 10^{-3}$. We note that the rate at which the dropping of $\mathrm{H}$ takes place on a grain is a very small number. To handle this, we drop one $\mathrm{H}$ after every $A_{\mathrm{H}} / F_{\mathrm{H}}$ number of hops. When $F_{\mathrm{H}}$ is very low, we continued our simulation for a larger time interval until a steady state is reached. Similarly, where $F_{\mathrm{H}}$ is large enough so that $F_{\mathrm{H}} / A_{\mathrm{H}}>1$, we drop several at each time step. In any case, for a given $F_{\mathrm{H}}$, the effective accretion rate is given by $\phi_{\mathrm{H}}=F_{\mathrm{H}}\left(1-f_{\text {grh }}-f_{\text {grh } 2}\right)$, where $f_{\text {grh }}$ and $f_{\text {grh } 2}$ are the fractions of the grain that are occupied by $\mathrm{H}$ and $\mathrm{H}_{2}$, respectively. After $1 / A_{\mathrm{H}}$ seconds, $\phi_{\mathrm{H}} / A_{\mathrm{H}}$ number of hydrogen atoms are dropped on the grain. The exact site at which one atom is dropped is obtained by a pair of random numbers $\left(R_{x}, R_{y} ; R_{x}<1\right.$ and $\left.R_{y}<1\right)$ obtained by a random number generator. This pair would place the incoming hydrogen at $(i, j)$ th grid, where $i$ and $j$ are the nearest integers obtained using the Int function: $i=\operatorname{int}\left(R_{x} * n+0.5\right)$ and $j=\operatorname{int}\left(R_{y} * n+0.5\right)$. Each atom starts hopping with equal probability in all four directions. This direction is decided by another random number. When, during the hopping process, one atom enters a site that is already occupied by another atom, we assume that a molecule has formed, and we increase the number of $\mathrm{H}_{2}$ by unity and reduce the number of $\mathrm{H}$ by two. However, when the atom enters a site occupied by an $\mathrm{H}_{2}$, another random number is generated to decide which of the other nearest sites it is going to occupy. Thermal evaporation of $\mathrm{H}$ and $\mathrm{H}_{2}$ from a grain surface are handled in the following way. Let $W_{\mathrm{H}}$ and $W_{\mathrm{H} 2}$ be the desorption coefficients of a hydrogen and hydrogen molecule given by $v \exp \left(-E_{1} / k_{\mathrm{b}} T\right)$ and $v \exp \left(-E_{2} / k_{\mathrm{b}} T\right)$, where $E_{1}$ and $E_{2}$ are the activation barrier energies (in $\mathrm{meV}$ ) for the desorption of $\mathrm{H}$ and the $\mathrm{H}_{2}$ molecule. Thus, one atom is released to the gas phase after every $1 / W_{\mathrm{H}} \mathrm{s}$, and one molecule is released after every $1 / W_{\mathrm{H} 2} \mathrm{~s}$. We generate a random number $R_{\mathrm{t}}$ for every $\mathrm{H}$ present on the grain, and release (at each time step, i.e., $1 / A_{\mathrm{H}} \mathrm{s}$ ) only those for which $R_{\mathrm{t}}<W_{\mathrm{H}} / A_{\mathrm{H}}$. A similar procedure is followed for the evaporation of $\mathrm{H}_{2}$, for which the criterion for evaporation was $R_{\mathrm{t}}<W_{\mathrm{H} 2} / A_{\mathrm{H}}$. There could also be spontaneous desorption and a factor of $1 \mu$ of $n_{\mathrm{H} 2}$ evaporated to the gas phase. Here too, a random number $R_{\mathrm{S}}$ is generated for each newly formed $\mathrm{H}_{2}$ present on the grain. Those that satisfy $R_{\mathrm{S}}<1-\mu$ are removed to the gas phase.

We continue our simulation for each type of grain and for each accretion rate and temperature for a duration more than $10^{6} \mathrm{~s}$ or more depending on the accretion rate $F_{\mathrm{H}}$, till a quasi-steady state is reached. After an initial transient period, $n_{\mathrm{H}}$ and $n_{\mathrm{H} 2}$, the number of $\mathrm{H}$ and $\mathrm{H}_{2}$ reach a quasi-steady state with some fluctuations. These numbers can be used to compute the recombination time. The values of the activation barrier energies $E_{0}, E_{1}$, and $E_{2}$ are taken from Katz et al. (1999) and are given by $E_{0}=24.7 \mathrm{meV}, E_{1}=32.1 \mathrm{meV}$, and $E_{2}=27.1 \mathrm{meV}$ for olivine and $E_{0}=44 \mathrm{meV}, E_{1}=56.7 \mathrm{meV}$, and $E_{2}=$ $46.7 \mathrm{meV}$ for amorphous carbon grains. For olivine, $\mu=0.33$, and for amorphous carbon, $\mu=0.413$ was used.

\section{Recombination timescale as a function of grain and gas parameters}

Using the results of our simulation, we are now in a position to compute a quantity $\alpha(t)$ defined by,

$\alpha(t)=\log \left(\frac{2 A_{\mathrm{H}} n_{\mathrm{H}}^{2}}{\phi_{\mathrm{H}}-W_{\mathrm{H}} n_{\mathrm{H}}}\right) / \log (S)$.

As $t \rightarrow \infty, \alpha(t) \rightarrow \alpha_{0}$. If there were no effect of the accretion rate and the finite size of the grain, then we would obtain $\alpha(t)=1$ identically at all the time. However, this is not what we find. In Figs. 1a,b, we plot $\alpha_{0}$ for amorphous carbon and olivine grains at $T=20 \mathrm{~K}$ and $T=10 \mathrm{~K}$, respectively, as a function of the accretion rate per site $a_{\mathrm{s}}$. For very very low rates, it becomes impossible to carry out the simulations in a computationally finite time. We thus extrapolated the curves for very low $a_{\mathrm{s}}$ values. We find that as the accretion rate per site goes down, $\alpha_{0}$ deviates from unity significantly. Thus, clearly, for theoretical considerations, the number of $\mathrm{H}$ and $\mathrm{H}_{2}$ should be calculated using Eq. (3b) and not Eq. (3a).

In this context, one important parameter $\beta$ may be defined as the "catalytic capacity" of a grain, which measures the efficiency 

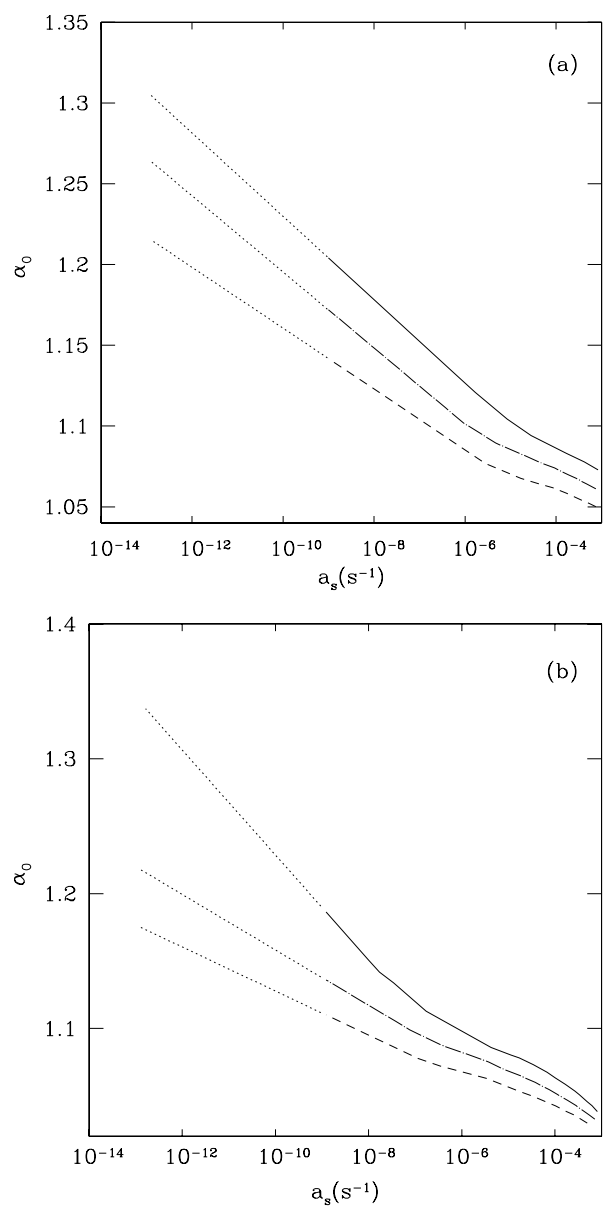

Fig. 1. a), b) Variation of $\alpha_{0}$ as a function of the accretion rate per site for an a) amorphous carbon grain kept at $20 \mathrm{~K}$ and b) olivine grain kept at $10 \mathrm{~K}$. The solid, dot-dashed, and dashed curves are for $10^{4}, 9 \times 10^{4}$, and $10^{6}$ sites, respectively. As the accretion rate is reduced, $\alpha_{0}$ starts to deviate from 1 as the effective grain site number goes up due to the sweeping of the grain more than once before recombination takes place. The deviation is highlighted using dotted curves by extrapolating at very low accretion rates.

of the formation of $\mathrm{H}_{2}$ on a grain surface for a given pair of $\mathrm{H}$ residing on it. Let $\delta N_{\mathrm{H} 2}$ be the number of $\mathrm{H}_{2}$ formed in $\delta t$ time. Since two hydrogen atoms are required to create one $\mathrm{H}_{2}$, the average rate of creation of one $\mathrm{H}_{2}$ per pair of $\mathrm{H}$ atom would be given by

$\left\langle A_{\mathrm{H} 1}\right\rangle=\frac{1}{2 n_{\mathrm{H}}} \frac{\delta N_{\mathrm{H}_{2}}}{\delta t}$.

We identify the inverse of this rate with the average formation rate given by

$T_{f}(t)=S^{\beta(t)} / A_{\mathrm{H}}$.

Thus,

$S^{\beta}(t)=A_{\mathrm{H}} /\left\langle A_{\mathrm{H} 1}\right\rangle$.

This yields $\beta(t)$ as a function of time as

$\beta=\log \left(A_{\mathrm{H}} /\left\langle A_{\mathrm{H} 1}\right\rangle\right) / \log (S)$.

Here too, we can define $\beta_{0}=\beta(t \rightarrow \infty)$. Our goal would be to see if $\beta_{0}$ thus obtained actually varies with grain parameters, accretion rate, and temperatures. We anticipated in Acharyya et al. (2005) that this exponent could be as low as 0.5 or less. Indeed,
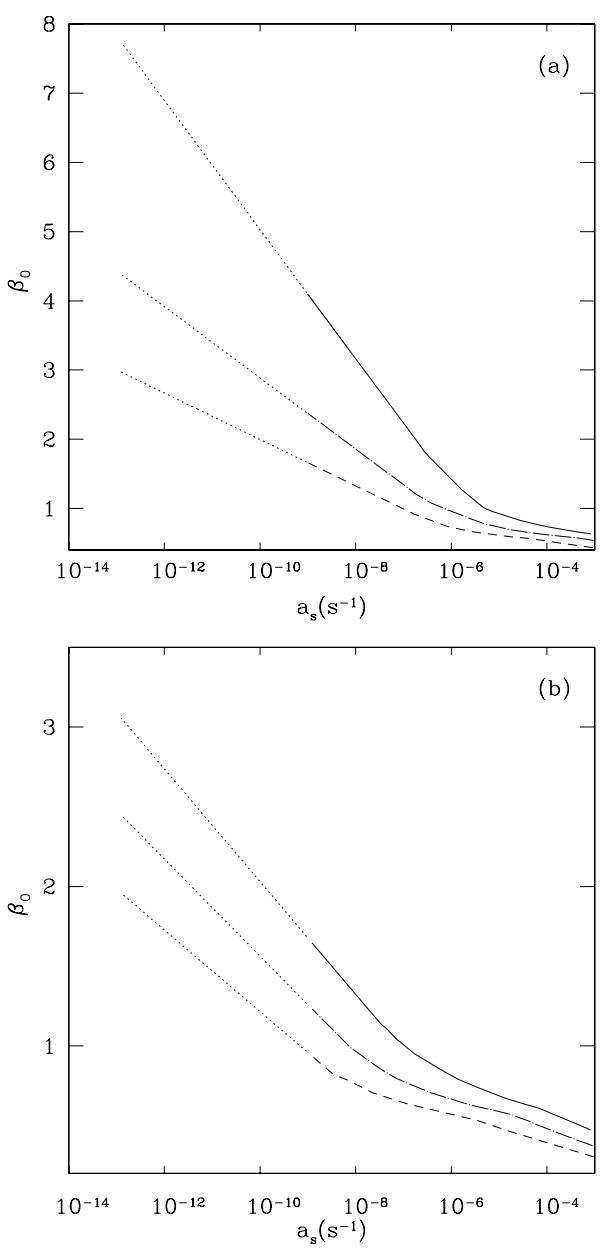

Fig. 2. a), b) Variation of $\beta_{0}$ as a function of the accretion rate per site for an a) amorphous carbon grain kept at $20 \mathrm{~K}$ and b) olivine grain kept at $10 \mathrm{~K}$. The solid, dot-dashed, and dashed curves are for $10^{4}$, $9 \times 10^{4}$, and $10^{6}$, sites respectively. As the accretion rate is reduced, $\beta_{0}$ becomes very high compared to unity, for the same reason as $\alpha_{0}$. As the accretion rate is increased, the grain becomes mostly occupied and an $\mathrm{H}$ has to hop an average of $S^{1 / 2}$ times before an $\mathrm{H}_{2}$ is produced. Thus, $\beta_{0}$ becomes almost $1 / 2$. The deviation is highlighted using dotted curves by extrapolating at very low accretion rates.

this is what we see as well. In Figs. $2 \mathrm{a}, \mathrm{b}$ we show $\beta_{0}$ as a function of the accretion rates per site for various grain, sites and for (a) amorphous carbon at $T=20 \mathrm{~K}$ and (b) olivine at $T=10 \mathrm{~K}$ grains, respectively. The solid, dotted, and dashed curves are for the $10^{4}, 9 \times 10^{4}$, and $10^{6}$ sites, respectively as before. We find that $\beta_{0}$ can vary anywhere between $\sim 1.5$ to $\sim 0.5$. Most interestingly, as the accretion rate becomes high, the exponent becomes close to 0.5 , as if the recombination is taking place on a onedimensional grain. This is perhaps understandable, since as the grain gets filled up, the maximum number of hopping in any direction should not be more than $n$, i.e., the number of sites in any one direction of the grain.

It is interesting to compare the results of our simulation with those obtained from the analytical considerations with and without our $\alpha_{0}$ factor in the equation mentioned above. In Table 1 , we present this comparison. We take an olivine grain of $10^{4}$ sites at $10 \mathrm{~K}$ and vary the accretion rates. In Col. 1, we give the accretion rate per site of the grain. In Col. 2 we present the coefficient $\alpha_{0}$ that we derive from our simulation. In Cols. 3-5, we present the number of $\mathrm{H}$ obtained by our simulation, the modified equation (Eq. (3b)), and the standard equations (Eq. (3a)). 
Table 1. Comparison of $\mathrm{H}$ and $\mathrm{H}_{2}$ abundances with various methods.

\begin{tabular}{cc|ccc|ccc}
\hline \hline Accretion rate & $\alpha_{0}$ & \multicolumn{3}{|c|}{$\mathrm{H}$ with } & \multicolumn{3}{c}{$\mathrm{H}_{2}$ with } \\
\cline { 3 - 8 } per site $A_{\mathrm{s}}\left(S^{-1}\right)$ & & Simulation & $\alpha_{0} \neq 1$ & $\alpha_{0}=1$ & Simulation & $\alpha_{0} \neq 1$ & $\alpha_{0}=1$ \\
$9.79 \times 10^{-3}$ & 0.9807 & 935.75 & 903.30 & 1008.83 & 1609.74 & 1581.09 & 1650.50 \\
\hline $8.58 \times 10^{-3}$ & 0.9857 & 909.51 & 884.82 & 958.35 & 1442.57 & 1447.73 & 1489.45 \\
$5.15 \times 10^{-3}$ & 1.0021 & 791.82 & 784.50 & 775.71 & 940.92 & 978.55 & 975.84 \\
$3.43 \times 10^{-3}$ & 1.0127 & 694.71 & 692.13 & 649.17 & 658.34 & 691.38 & 683.45 \\
$2.58 \times 10^{-3}$ & 1.0191 & 627.84 & 626.46 & 569.76 & 507.21 & 533.68 & 526.46 \\
$1.71 \times 10^{-3}$ & 1.0271 & 539.40 & 538.32 & 471.95 & 349.42 & 366.19 & 361.22 \\
$8.58 \times 10^{-4}$ & 1.0385 & 408.56 & 407.07 & 339.14 & 180.06 & 188.55 & 186.52 \\
$1.72 \times 10^{-4}$ & 1.0586 & 203.73 & 202.09 & 154.06 & 37.40 & 38.62 & 38.49 \\
$1.72 \times 10^{-5}$ & 1.0783 & 71.38 & 69.68 & 48.75 & 3.72 & 3.83 & 3.85 \\
$1.37 \times 10^{-6}$ & 1.1013 & 21.41 & 21.10 & 13.49 & 0.28 & 0.280 & 0.2951 \\
$1.72 \times 10^{-7}$ & 1.1170 & 7.32 & 7.20 & 4.49 & 0.027 & 0.0290 & 0.0327 \\
$1.14 \times 10^{-7}$ & 1.1211 & 5.89 & 5.76 & 3.59 & 0.018 & 0.018 & 0.0209 \\
$3.43 \times 10^{-8}$ & 1.1329 & 2.85 & 2.78 & 1.79 & 0.0038 & 0.0037 & 0.0052 \\
\hline
\end{tabular}

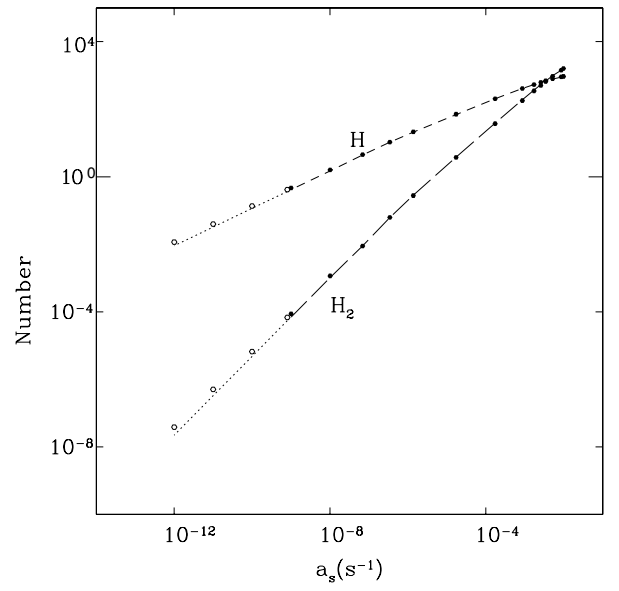

Fig. 3. Comparison of the simulation results (dark circles) with those obtained from analytical considerations (Eq. (2a,b)) dashed curves) when suitable modification of the average recombination rate (Eq. (3b)) is made. An olivine grain of $10^{4}$ sites at a temperature of $10 \mathrm{~K}$ has been chosen in this comparison. Dotted curves are drawn using analytical results for $\alpha_{0}$ extrapolated to very low accretion rates.

Columns 6-8, we present similar results for $\mathrm{H}_{2}$. We find that our simulation matches with the analytical results more accurately provided Eq. (3b) is chosen. If, the standard equation (Eq. (3a)) is used, the deviation is very significant.

In Fig. 3, we compare the number of $\mathrm{H}$ and $\mathrm{H}_{2}$ on an olivine grain of $10^{4}$ sites kept at $10 \mathrm{~K}$ as a function of the accretion rate per site. The dashed curves are drawn using Eqs. (2a,b), and (3b) (using $\alpha_{0}$ from the simulation), while the dark circles are purely from our simulation. Using extrapolated $\alpha_{0}$ (from Fig. 1b), we computed $n_{\mathrm{H}}$ and $n_{\mathrm{H} 2}$ analytically and, see very good agreements with the simulation at lower rates.

\section{Concluding remarks}

In this paper, we carried out a numerical simulation to show that the effective grain surface is not really the same as the physical grain surface. The simulations have been carried out for amorphous carbon and olivine grains kept at $20 \mathrm{~K}$ and $10 \mathrm{~K}$, respectively, for a wide range of accretion rates. In the literature, it is assumed that the recombination time is the grain site number divided by the diffusion rate (Eq. (3a)). However, we find that this simplistic assumption is not valid, especially when the accretion rate of $\mathrm{H}$ on the grains is very low. In this case, an atomic hydrogen may have to sweep a grain several times before meeting another atom to form a molecule, thereby increasing the effective surface area. For very high rates, the grain surfaces could be partially filled with $\mathrm{H}$ and $\mathrm{H}_{2}$, and the search for another $\mathrm{H}$ need not take $S$ number of hops. We found that for an average, $\alpha_{0}$, the index that determines the effective number of sites could be $\gtrsim 1$ for very low rates. We also find that $\alpha_{0}$ depends on the nature of the grains, the temperature, and the grain site numbers. Thus, the recombination time is a complex function of these parameters. From the simulation results, we defined another in$\operatorname{dex} \beta$, which is a measure of the average catalytic ability to form an $\mathrm{H}_{2}$. This index is also found to be a strong function of the accretion rate. In fact, for very high rates, the index may go down to 0.5, as anticipated in ACC05. A comparison of the recombination efficiency, as obtained from our procedure, with that obtained from the standard consideration (Eq. (1a,b)) clearly shows a deviation easily attributable to the site/rate dependence of $\alpha_{0}$ in a realistic case. The considerations based on which we came to our conclusion are so generic that we believe that similar dependence should follow even for other grain surface reactions. This will be discussed elsewhere. We believe that this important behaviour will have to be kept in mind in future studies of chemical evolution in interstellar clouds in the presence of grains.

Acknowledgements. The work of A.D. has been supported by a DST Project and that of K.A. is supported by an ISRO Project with the Centre for Space Physics.

\section{References}

Acharyya, K., \& Chakrabarti, S. K. 2005, Bul. Astron. Soc. India, 33, 473 Acharyya, K., Chakrabarti, S. K., \& Chakrabarti, S. 2005, MNRAS, 361, 550 Biham, O., Furman, I., Pirronello, V., \& Vidali, G. 2001, ApJ, 553, 595 Cazaux, S., \& Tielens, A. G. G. M. 2002, ApJ, 575, L29 Cazaux, S., \& Tielens, A. G. G. M. 2004, ApJ, 604, 222

Green, N. J. B, Toniazzo, T., Pilling, M. J., et al. 2001, A\&A, 375, 1111 Hollenbach, D., Werner, M. W., \& Salpeter, E. E. 1971, ApJ, 163, 165 Katz, N., Furmann, I., Biham, O., Pironello, V., \& Vidali, G. 1999, ApJ, 522, 305 Pirronello, V., Liu, C., Riser, J. E., \& Vidali, G. 1999, A\&A, 344, 681 Rae, J. G. L., Green, N. J. B., Hartquist, T. W., Pilling, M. J., \& Toniazzo, T. 2003, A\&A, 405, 387

Takahashi, J., Matsuda, K., \& Nagaoka, M. 1999, ApJ, 520, 724 